\title{
A Unified Topological Framework for Digital Imaging
}

\author{
Loïc Mazo $^{1,2, \star}$, Nicolas Passat ${ }^{1}$, Michel Couprie $^{2}$, and Christian Ronse ${ }^{1}$ \\ ${ }^{1}$ Université de Strasbourg, LSIIT, UMR CNRS 7005, France \\ ${ }^{2}$ Université Paris-Est, Laboratoire d'Informatique Gaspard-Monge, Équipe A3SI, \\ ESIEE Paris, France \\ \{loic.mazo, passat, cronse\}@unistra.fr, m.couprie@esiee.fr
}

\begin{abstract}
In this article, a tractable modus operandi is proposed to model a (binary) digital image (i.e., an image defined on $\mathbb{Z}^{n}$ and equipped with a standard pair of adjacencies) as an image defined in the space of cubical complexes $\left(\mathbb{F}^{n}\right)$. In particular, it is shown that all the standard pairs of adjacencies in $\mathbb{Z}^{n}$ can then be correctly modelled in $\mathbb{F}^{n}$. Moreover, it is established that the digital fundamental group of a digital image in $\mathbb{Z}^{n}$ is isomorphic to the fundamental group of its corresponding image in $\mathbb{F}^{n}$, thus proving the topological correctness of the proposed approach. From these results, it becomes possible to establish links between topology-oriented methods developed either in classical digital spaces $\left(\mathbb{Z}^{n}\right)$ or cubical complexes $\left(\mathbb{F}^{n}\right)$.
\end{abstract}

Keywords: digital imaging, digital topology, cubical complexes, homotopy, fundamental group.

\section{Introduction}

The rise of digital imaging, and the associated need of efficient image analysis tools, have provided a strong motivation to research related to the definition of sound digital topological models. Indeed, in order to process digital images, it is often fundamental to be able to preserve, get back or integrate topological information.

Basically, an $n$-dimensional (digital) binary image can be considered as a subset of $\mathbb{Z}^{n}$. However, the actual structures visualised in such images are generally continuous ones, corresponding to objects of the real world, i.e., objects of $\mathbb{R}^{n}$, and not of $\mathbb{Z}^{n}$. In order to deal with this continuous/discrete issue, research efforts have essentially focused on specific and pragmatic questions related to topology, namely the definition of a notion of adjacency relation, and the induced notions of connectivity and arcs. These notions lead, in particular, to high-level concepts of topology, such as homotopy, with natural applications to "homotopy type-preserving" image processing.

The first solution proposed to model the topology of a digital image in $\mathbb{Z}^{n}$ was to consider that two points (also called xels) are adjacent if they are neighbours in the $n$-D cubic grid naturally induced by $\mathbb{Z}^{n}$. In this framework, partial solutions have been found to model as well as possible the above topological properties, for instance by defining dual adjacencies for the object and the background (composed of the xels of value

\footnotetext{
* The research leading to these results has received funding from the French Agence Nationale
} de la Recherche (Grant Agreement ANR-2010-BLAN-0205). 
1 and 0 , respectively), enabling to define, from these adjacency relations, the notions of connectivity [19] and of digital fundamental group [10], which permits to compare objects from a topological point of view. This approach is known as digital topology [13].

Other discrete spaces, enabling to model the continuous topological properties of digital images, have also been proposed. These alternative approaches of topology modelling are connected ordered topological spaces [9], abstract cell complexes [14] and orders [4]. Broadly speaking, they propose to put some "topological glue" between the xels of digital images to define the topological links with their continuous analogues.

By comparison to these (more sophisticated) approaches, digital topology may appear as the less satisfactory solution to deal with topological properties of binary images. Nevertheless, it remains the most commonly used framework for developing image processing tools dealing with topological issues. Indeed, since digital topology is directly defined in $\mathbb{Z}^{n}$, methods relying on it will also provide final results in $\mathbb{Z}^{n}$, which is a desired property in most applications. Moreover, a large literature has been devoted to homotopy-type preservation in digital topology, especially thanks to the concept of simple point [6]. In this context, very few methods have been based on alternative models while digital topology has led to the design of quite numerous ones (see, e.g., [17]).

Because of this intensive use of digital topology, it may be important to guarantee that there exists an actual compatibility between digital topology and the other proposed topological approaches (and more generally with the "continuous" topology). This requires to be able to embed a binary image defined in $\mathbb{Z}^{n}$ into a richer space (while respecting the chosen adjacencies in $\mathbb{Z}^{n}$ ) while preserving certain topological characteristics of objects (see e.g. [11[162 158]).

The "richer space" that is used here is $\mathbb{F}^{n}$, namely the space of cubical complexes, which is together a connected ordered topological space, a cellular space and an order (i.e., a poset). Though it is commonly admitted that there exists a strong link between digital topology and cubical complexes [12], since complexes are closed objects, the images handled in $\mathbb{F}^{n}$ correspond generally to images defined in $\mathbb{Z}^{n}$ with a $\left(3^{n}-1,2 n\right)$ adjacency pair. In [5], a method has been proposed to retrieve and improve digital topology in the framework of posets, but the case of the $(6,18)$ - and $(18,6)$-adjacency pairs was not considered. In [3], a way is described to embed digital pictures in a space of complexes according to the kind of connectivity chosen in $\mathbb{Z}^{n}$. However, there is no use of an intrinsic topology on complexes which are just a step between $\mathbb{Z}^{n}$ and $\mathbb{R}^{n}$, leading to define specific notions of connectedness and digital homotopy in $\mathbb{F}^{n}$. Thereby, in this paper, we propose a complete framework to correctly embed a binary digital image in the topological space $\mathbb{F}^{n}$, according to the choice of adjacencies which has been made in $\mathbb{Z}^{n}$.

The article is organised as follows. Sec. 2 recalls background notions. Sec. 3 describes the mapping enabling to associate a binary image defined on $\mathbb{Z}^{n}$ to an equivalent image defined in $\mathbb{F}^{n}$. Sec. 4 presents the main contribution of this work. It states that the connected components and the digital fundamental group of the digital images in $\mathbb{Z}^{n}$ are preserved in $\mathbb{F}^{n}$ when using the mapping described in Sec. 3 . Sec. 5 concludes this article. By lack of space, no proofs are given in this article, they can be found in [17]. 


\section{Background Notions}

This section provides the minimal set of background notions required to make this article globally self-contained, and then more comprehensible for the reader.

\subsection{Posets}

A partially ordered set (or poset) is a ordered pair $(X, \leqslant)$ where $X$ is a set and the relation $\leqslant$ is a partial order on $X$. We write $x<y$ when $x \leqslant y$ and $x \neq y$. The relation $\geqslant$ defined on $X$ by $x \geqslant y$ if $y \leqslant x$ is a partial order on $X$ called the dual order. The covering relation $\prec$, associated to $\leqslant$, is defined by: $x<y$ (say " $y$ covers $x$ ") if $x<y$ and there is no $z$ such that $x<z<y$. We set: $x^{\uparrow}=\{y \in X \mid x \leqslant y\} ; x^{\uparrow+}=\left\{y \in x^{\uparrow} \mid y^{\uparrow}=\{y\}\right.$; $x^{\downarrow}=\{y \in X \mid y \leqslant x\} ; x^{\downarrow \star}=x^{\downarrow} \backslash\{x\}=\{y \in X \mid y<x\} ; x^{\prec}=\{y \in X \mid x<y\}$. An element $x \in X$ is minimal if $x^{\downarrow}=\{x\}$ and maximal if $x^{\uparrow}=\{x\}$. An element $x \in X$ is the minimum of $X$ if $x^{\uparrow}=X$ and is the maximum of $X$ if $x^{\downarrow}=X$. If the minimum (resp., the maximum) exists, then it is unique.

\subsection{Topological Spaces}

Let $(X, \leqslant)$ be a poset. The set $\mathcal{U}$ defined by $\mathcal{U}=\left\{U \subseteq X \mid \forall x \in U, x^{\uparrow} \subseteq U\right\}$ is a topology on $X$ which is called the Alexandroff topology. In this topology, the set $x^{\uparrow}$ is the smallest open set containing $x$ (or the smallest neighbourhood of $x$, called the star of $x$ ) and the set $x^{\downarrow}$ is the smallest closed set containing $x$ (the closure of $x$ ). Two points $x, y \in X$ are adjacent if $x \leqslant y$ or $y \leqslant x$. A sequence $\left(z_{i}\right)_{i=0}^{r}(r \geq 0)$ of elements of $X$ is an arc in $X$ (from $z_{0}$ to $z_{r}$ ) if for all $i \in \llbracket 1, r \rrbracket \mathbb{1}, z_{i-1}$ and $z_{i}$ are distinct and adjacent. A subset $Y$ of $X$ is connected if for all $x, y \in Y$, there exists an arc in $Y$ from $x$ to $y$. A connected component of a subset $Y$ of $X$ is a maximal (for inclusion) connected subset of $Y$.

The closure $Y^{\downarrow}$ of a subset $Y \subseteq X$ is the smallest closed set including $Y$. The interior $Y^{\circ}$ of a subset $Y \subseteq X$ is the largest open set included in $Y$. Closure and interior are dual notions since $\neg\left(Y^{\circ}\right)=(\neg Y)^{\downarrow}$ and $\neg\left(Y^{\downarrow}\right)=(\neg Y)^{\circ}$ where $\neg Y=X \backslash Y$. An open set $Y$ is a regular open set if $Y=\left(Y^{\downarrow}\right)^{\circ}$ and a closed set is a regular closed set if $Y=\left(Y^{\circ}\right)^{\downarrow}$. The complement of a regular open set is a regular closed set.

\subsection{Cubical Complexes}

Let $\mathbb{Z}$ be the set of integers. Let $\mathbb{F}_{0}^{1}=\{\{a\} \mid a \in \mathbb{Z}\}$ and $\mathbb{F}_{1}^{1}=\{\{a, a+1\} \mid a \in \mathbb{Z}\}$. Let $n \geq 1$.

Let $f \subset \mathbb{Z}^{n}$. If $f$ is the Cartesian product of $m$ elements of $\mathbb{F}_{1}^{1}$ and $n-m$ elements of $\mathbb{F}_{0}^{1}$, we say that $f$ is a face or an $m$-face $\left(\right.$ of $\mathbb{Z}^{n}$ ), $m$ is the dimension of $f$, and we write $\operatorname{dim}(f)=m$ (some faces of $\mathbb{Z}^{2}$ are depicted in Fig. 1). We denote by $\mathbb{F}^{n}$ the set composed of all faces of $\mathbb{Z}^{n}$; this set is the (n-D) space of cubical complexes. We denote by $\mathbb{F}_{k}^{n}(0 \leq k \leq n)$ the set composed of all $k$-faces of $\mathbb{Z}^{n}$. The couple $\left(\mathbb{F}^{n}, \subseteq\right)$ is a poset. Let $F \subseteq \mathbb{F}^{n}$ be a set of faces. Let $f \in F$ be a face. The face $f$ is a facet of $F$ if $f$ is maximal in $F$. In particular, if $x=\left(x_{i}\right)_{i=1}^{n} \in \mathbb{Z}^{n}$, the set $\dot{x}=\prod_{i=1}^{n}\left\{x_{i}, x_{i}+1\right\}$ is a facet of $\mathbb{F}^{n}$.

\footnotetext{
${ }^{1}$ We write $\llbracket p, q \rrbracket$ for an integer interval and $[p, q]$ for a real interval.
} 


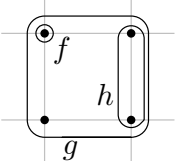

(a)

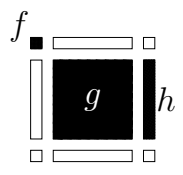

(b)

Fig. 1. Two representations of a set of faces $F=\{f, g, h\}$ in $\mathbb{Z}^{2}$ with, for instance, $f=\{0\} \times\{1\}$, $g=\{0,1\} \times\{0,1\}$ and $h=\{1\} \times\{0,1\}$. The face $g$ is a facet of $F\left(\right.$ and also of $\left.\mathbb{F}^{2}\right)$.

\subsection{Digital Topology}

Let $x=\left(x_{i}\right)_{i=1}^{n}$ and $y=\left(y_{i}\right)_{i=1}^{n}$ be two points in $\mathbb{Z}^{n}$ (also called $n$-xels, or simply xels). The points $x$ and $y$ are $2 n$-adjacent if $\sum_{i=1}^{n}\left|x_{i}-y_{i}\right|=1$. They are $\left(3^{n}-1\right)$-adjacent if $\max _{i=1}^{n}\left\{\left|x_{i}-y_{i}\right|\right\}=1$. When $n=3$, the points $x$ and $y$ are 18-adjacent if they are 26adjacent and $\sum_{i=1}^{n}\left|x_{i}-y_{i}\right| \leq 2$. Let $\alpha \in\left\{2 n, 3^{n}-1\right\}$ (or possibly $\alpha=18$ if $n=3$ ). Any point in $\mathbb{Z}^{n}$ is $\alpha$-adjacent to $\alpha$ other points. A sequence $\gamma=\left(z_{i}\right)_{i=0}^{r}(r \geq 0)$ of points in $X \subseteq \mathbb{Z}^{n}$ is a (digital) $\alpha$-path (from $z_{0}$ to $z_{r}$ ) if for all $i \in \llbracket 1, r \rrbracket, z_{i-1}$ and $z_{i}$ are $\alpha$-adjacent. The integer $r$ is the length of $\gamma$. A subset $X \subseteq \mathbb{Z}^{n}$ is $\alpha$-connected, if for all $x, y \in X$, there exists a digital $\alpha$-path from $x$ to $y$ in $X$. In order to retrieve some topological features in binary digital images (such as the notion of hole), it is necessary to use pairs of adjacencies, one for the object $X$ and one for the background $\mathbb{Z}^{n} \backslash X$. The suitable pairs are $\left(2 n, 3^{n}-1\right)$ and $\left(3^{n}-1,2 n\right)$ (plus, when $n=3,(6,18)$ and $\left.(18,6)\right)$.

\section{Connectivity: From $\mathbb{Z}^{n}$ to $\mathbb{\mathbb { N }}^{n}$}

A (digital) image $\lambda$ on $\mathbb{Z}^{n}$ is a function from $\mathbb{Z}^{n}$ to $\{0,1\}$. A (complex) image $\mu$ on $\mathbb{F}^{n}$ is a function from $\mathbb{F}^{n}$ to $\{0,1\}$. The object (resp. the background) associated to the image $\theta: X \rightarrow\{0,1\}$ (with $X=\mathbb{Z}^{n}$ or $\left.\mathbb{F}^{n}\right)$ is the set $\theta^{-1}(\{1\})\left(\right.$ resp. $\theta^{-1}(\{0\})$ ).

If $\mu$ is a complex image, we write $\bigvee_{x \in X} \mu(x)$ (resp. $\bigwedge_{x \in X} \mu(x)$ ) for the maximum (resp. minimum) of the set $\{\mu(x) \mid x \in X\}$ and we also write $\mu(a) \vee \mu(b)$ (resp. $\mu(a) \wedge \mu(b))$ for $\bigvee_{x \in\{a, b\}} \mu(x)\left(\right.$ resp. $\left.\bigwedge_{x \in\{a, b\}} \mu(x)\right)$.

The poset $\left(\mathbb{F}^{n}, \subseteq\right)$ is equipped with its Alexandroff topology.

\subsection{One-to-One Correspondence between Images on $\mathbb{Z}^{n}$ and $\mathbb{F}^{n}$}

When two faces $g, h \in \mathbb{F}^{n}$ cover a face $f \in \mathbb{F}^{n}$ and their smallest neighbourhoods do not intersect (i.e., $g^{\uparrow} \cap h^{\uparrow}=\emptyset$ ), we say that they are opposite with respect to the face $f$. We denote $\operatorname{opp}(f)$ the set of all $\{g, h\}$ for $g$ opposite to $h$ w.r.t. $f$. Intuitively, the face $f$ is required to "locally connect" the faces $g$ and $h$. When $f$ is a facet, we have opp $(f)=\emptyset$.

Definition 1. (regular image) Let $\varepsilon: \llbracket 1, n \rrbracket \rightarrow\{-1,1\}$ be a function called connectivity function 2 function $\mu: \mathbb{F}^{n} \rightarrow\{0,1\}$ is an $\varepsilon$-regular image (or simply a regular image) if for all $m \in \llbracket 1, n \rrbracket$ and $f \in \mathbb{F}_{m-1}^{n}$, we have, recursively

\footnotetext{
${ }^{2}$ We write $\varepsilon=\left(a_{1}, \ldots, a_{n}\right)$ for the function $\varepsilon$ such that $\varepsilon(i)=a_{i}$ for all $i \in \llbracket 1, n \rrbracket$, as done for instance in Fig. 2] We also write $\varepsilon=1$ (or $\varepsilon=-1$ ) when $\varepsilon$ is a constant function.
} 


$$
\mu(f)= \begin{cases}\bigwedge_{\{a, b\} \in \operatorname{opp}(f)} \mu(a) \vee \mu(b) & \text { if } \varepsilon(m)=1 \\ \bigvee_{\{a, b\} \in \operatorname{opp}(f)} \mu(a) \wedge \mu(b) & \text { if } \varepsilon(m)=-1\end{cases}
$$

For each connectivity function $\varepsilon: \llbracket 1, n \rrbracket \rightarrow\{-1,1\}$, we define the function $\zeta_{\varepsilon}:$ $\{0,1\}^{\mathbb{Z}^{n}} \rightarrow\{0,1\}^{\mathbb{F}^{n}}$ which maps any digital image $\lambda$ to the unique $\varepsilon$-regular image $\zeta_{\varepsilon}(\lambda)$ such that, for each $a \in \mathbb{Z}^{n}$, we have $\zeta_{\varepsilon}(\lambda)(\dot{a})=\lambda(a)$. It is obvious that, for each $\varepsilon$, the function $\zeta_{\varepsilon}$ is a bijection between the set of digital images $\{0,1\}^{\mathbb{Z}^{n}}$ and the subset of $\varepsilon$-regular images of $\{0,1\}^{\mathbb{F}^{n}}$. Moreover, thanks to the choice of the connectivity function $\varepsilon$, we can accurately "carve" an image in $\mathbb{F}^{n}$ to model the desired connectivity in $\mathbb{Z}^{n}$ (see Fig. 2). In particular, we can get the usual pairs of adjacencies (see Figs. 2, 4 and Table 1). In Section 4, the correspondences given in Table 1 will be justified by two theorems establishing that, by following these links, we preserve the connected components and fundamental groups.

When the function $\varepsilon$ is constant, Definition 1 can be simplified. Note that the case $\varepsilon=-1$ corresponds to the $2 n$-adjacency in $\mathbb{Z}^{n}$ while the case $\varepsilon=1$ corresponds to the $\left(3^{n}-1\right)$-adjacency in $\mathbb{Z}^{n}$.

Proposition 1. Let $\mu: \mathbb{F}^{n} \rightarrow\{0,1\}$ be an $\varepsilon$-regular image. Let $f$ be a face of $\mathbb{F}^{n}$.

(i) If $\forall m>\operatorname{dim}(f), \varepsilon(m)=-1$, then we have $\mu(f)=\bigwedge_{f<a} \mu(a)=\bigwedge_{a \in f^{\uparrow+}} \mu(a)$

(ii) If $\forall m>\operatorname{dim}(f), \varepsilon(m)=1$, then we have $\mu(f)=\bigvee_{f<a} \mu(a)=\bigvee_{a \in f^{\uparrow+}} \mu(a)$

In particular, if $\varepsilon=-1$ then $\mu(f)=\bigwedge_{a \in f^{\uparrow+}} \mu(a)$ for all $f \in \mathbb{F}^{n}$, and if $\varepsilon=1$ then $\mu(f)=\bigvee_{a \in f^{\uparrow+}} \mu(a)$ for all $f \in \mathbb{F}^{n}$.

\subsection{Duality}

Let $\theta: X \rightarrow\{0,1\}$ with $\left(X=\mathbb{Z}^{n}\right.$ or $\left.\mathbb{F}^{n}\right)$ be an image. We define the negative image $\neg \theta: X \rightarrow\{0,1\}$ of $\theta$ by $\neg \theta(x)=1-\theta(x)$, for all $x \in X$.

Proposition 2. If $\mu: \mathbb{F}^{n} \rightarrow\{0,1\}$ is an $\varepsilon$-regular image, then $\neg \mu$ is $(-\varepsilon)$-regular.

Let $\mu: \mathbb{F}^{n} \rightarrow\{0,1\}$ be an $\varepsilon$-regular image. We define the image $-\mu: \mathbb{F}^{n} \rightarrow\{0,1\}$ by $(-\mu)(f)=\mu(f)$ for all $f \in \mathbb{F}_{n}^{n}$ and $\mu$ is $(-\varepsilon)$-regular.

Proposition 3. If $\mu: \mathbb{F}^{n} \rightarrow\{0,1\}$ is an $\varepsilon$-regular image, then we have $\neg(-\mu)=-(\neg \mu)$.

From the above definitions and propositions, we straightforwardly derive the following result.

Proposition 4. Let $\lambda: \mathbb{Z}^{n} \rightarrow\{0,1\}$ be a digital image. Let $\varepsilon: \llbracket 1, n \rrbracket \rightarrow\{-1,1\}$ be a connectivity function. Then, we have $\neg\left(\zeta_{\varepsilon}(\lambda)\right)=\zeta_{-\varepsilon}(\neg \lambda)$.

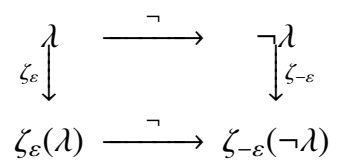




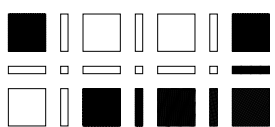

(a) $\varepsilon=(-1,-1)$

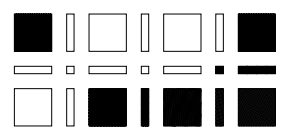

(b) $\varepsilon=(1,-1)$

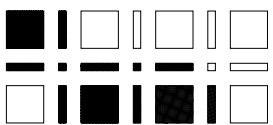

(c) $\varepsilon=(-1,1)$

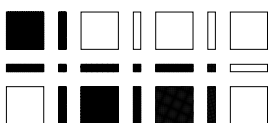

(d) $\varepsilon=(1,1)$

Fig. 2. Images $\zeta_{\varepsilon}(\lambda): \mathbb{F}^{2} \rightarrow\{0,1\}$ for some given $\lambda: \mathbb{Z}^{2} \rightarrow\{0,1\}$

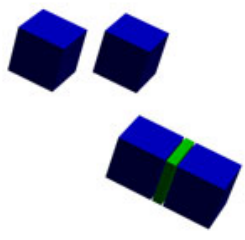

(a)

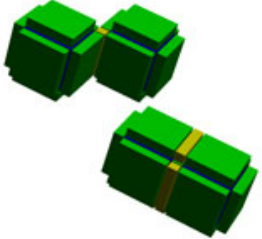

(b)

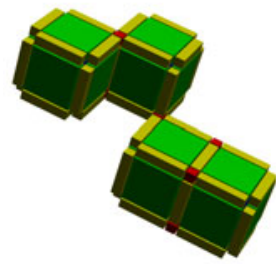

(c)

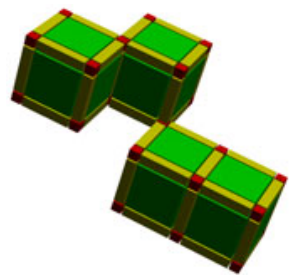

(d)

Fig. 3. Images $\zeta_{\varepsilon}(\lambda): \mathbb{F}^{3} \rightarrow\{0,1\}$ for some given $\lambda: \mathbb{Z}^{3} \rightarrow\{0,1\}$. In Figs. 34 the different colours are only used to distinguish the faces of the object $\left(\zeta_{\varepsilon}(\lambda)\right)^{-1}(\{1\})$ (blue: 3 -faces; green: 2-faces; yellow: 1-faces; red: 0-faces). (a) With $\varepsilon(3)=-1$ (whatever values for $\varepsilon(1)$ and $\varepsilon(2)$ ), we obtain the 6-adjacency (for the object) in $\mathbb{Z}^{3}$. (b) With $\varepsilon(3)=1$ and $\varepsilon(2)=-1$, we obtain the 18 -adjacency in $\mathbb{Z}^{3}$. (c, d) With $\varepsilon(3)=\varepsilon(2)=1$, we obtain the 26-adjacency in $\mathbb{Z}^{3}$.

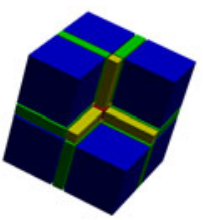

(a)

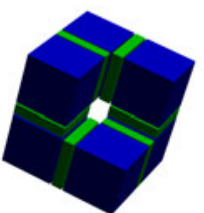

(b)

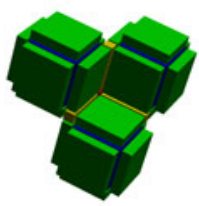

(c)

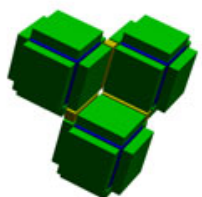

(d)

Fig. 4. A torus built with six 3-faces illustrates how the two 6-adjacencies can be obtained. (a) With $\varepsilon=( \pm 1,1,-1)$, the foreground is a horn-torus so we obtain the 6-adjacency associated to the 18-adjacency. (b) With $\varepsilon=( \pm 1,-1,-1)$, the foreground is a ring-torus, so we obtain the 6-adjacency associated to the 26-adjacency. (c,d) An object built from three facets with two connectivity functions which could a priori be used to model the 18-adjacency (see Fig. 3 b)). In (c), with $\varepsilon=(1,-1,1)$, we can see a red 0 -face between the three cubes. This is what is expected for the background must have a 6 -adjacency. In (d), with $\varepsilon=(-1,-1,1)$, there is a hole instead of the red 0 -face, which is not correct in 18-adjacency.

Table 1. Correspondence between pairs of adjacencies in $\mathbb{Z}^{n}$ and connectivity functions

\begin{tabular}{|c|c|c|c|c|c|c|c|c|}
\hline Space dimension & \multicolumn{2}{|c|}{$n=2$} & \multicolumn{5}{|c|}{$n=3$} & \multicolumn{2}{c|}{$n \geq 4$ (actually, $\left.n \in \mathbb{N}^{*}\right)$} \\
\hline Adjacencies in $\mathbb{Z}^{n}$ & $(4,8)$ & $(8,4)$ & $(6,26)$ & $(6,18)$ & $(18,6)$ & $(26,6)$ & $\left(2 n, 3^{n}-1\right)$ & $\left(3^{n}-1,2 n\right)$ \\
\hline$\varepsilon$ & -1 & 1 & -1 & $(-1,1,-1)$ & $(1,-1,1)$ & 1 & -1 & 1 \\
\hline
\end{tabular}




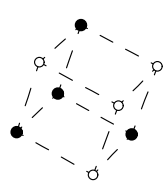

(a)

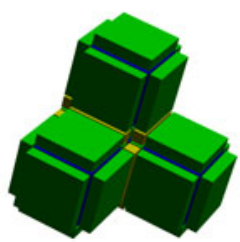

(b) $\varepsilon=(-1,-1,1)$

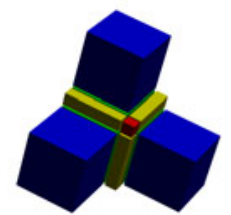

(c) $\varepsilon=(1,1,-1)$

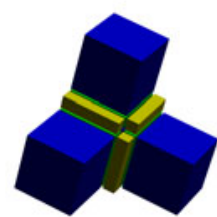

(d) $\varepsilon=(-1,1,-1)$

Fig. 5. (a) Symbolic representation of a trihedron related to a face $f \in \mathbb{F}^{n}$ such that $\operatorname{dim}(f)=n-3$. Black dots: $f_{1}^{\uparrow+}$; white dots $f^{\uparrow+} \backslash f_{1}^{\uparrow+}$. The dash lines represent the existence of a face of dimension $n-1$ forming the intersection between two facets of $f^{\uparrow+}$. (b-d) Examples of trihedra, with three connectivity functions $\varepsilon$ (one of the blue facets is hidden).

Remark 1. This proposition establishes that, for a given connectivity function $\varepsilon$ (and the associated pair of adjacencies $(\alpha, \beta))$, all the properties valid for $\lambda^{-1}(\{1\})$ and $\mu^{-1}(\{1\})$ are also valid for $\lambda^{-1}(\{0\})$ and $\mu^{-1}(\{0\})$ for the opposite connectivity function $-\varepsilon$ (and the associated pair of adjacencies $(\beta, \alpha))$. Broadly speaking, this means that the notions of object and background can be switched without loss of generality, provided that the pair of adjacencies $(\beta, \alpha)$ is also switched accordingly.

\subsection{Computing Values Directly from Facets}

The aim of this section is to find the number of facets which must have the value 1 in the star of a face to ensure that this face also has value 1 . In $\mathbb{F}^{2}$, the answer is straightforward. In $\mathbb{F}^{3}$, it requires to carefully study a particular configuration (depicted in Fig. 5), however, it can be answered, as stated hereafter. In higher dimensional spaces, the particular configurations to study are too numerous to get a useful result.

Let $f \in \mathbb{F}^{n}$, with $n \geq 3$. If $\operatorname{dim}(f)=n-3$, the poset $\left(f^{\uparrow}, \subseteq\right)$ has a unique minimum, namely $f$, and 8 maximal elements, namely the facets forming $f^{\uparrow+}$. From an adjacency point of view, these facets are geometrically organised as the 8 vertices of a cubical structure. When $f_{1}^{\uparrow+}$ (i.e., the facets of $f^{\uparrow+}$ whose values are equal to 1 ) is organised as in the configuration depicted in Fig. 5(a) (up to rotations and symmetries), we say that $f_{1}^{\uparrow+}$ is a trihedron. We define $\operatorname{Card}^{-}(E)=3$ and $\operatorname{Card}^{+}(E)=5$, if $E$ is a trihedron, and $\operatorname{Card}^{-}(E)=\operatorname{Card}^{+}(E)=\operatorname{Card}(E)$ otherwise.

For each connectivity function $\varepsilon$, we define recursively the function $\delta_{\varepsilon}: \llbracket 0, n \rrbracket \rightarrow$ $\llbracket 1,2^{n} \rrbracket$ by $\delta_{\varepsilon}(0)=1$, and for all $i>0, \delta_{\varepsilon}(i+1)=2 \delta_{\varepsilon}(i)-1$ if $\varepsilon(n-i)=1$, and $\delta_{\varepsilon}(i+1)=2 \delta_{\varepsilon}(i)$ if $\varepsilon(n-i)=-1$. It is easy to check that, for all $m \in \llbracket 0, n \rrbracket$, we have $\delta_{\varepsilon}(m)=1+\sum_{k=1}^{m}(1-\varepsilon(n-k+1)) 2^{m-k-1}$.

In the sequel, we write $f_{1}^{\uparrow+}$ for the set of facets in the star of $f$ which have value $1: f_{1}^{\uparrow+}=\left\{g \in \mathbb{F}_{n}^{n} \mid f \leqslant g\right.$ and $\left.\mu(g)=1\right\}$.

Proposition 5. Let $\mu: \mathbb{F}^{n} \rightarrow\{0,1\}$ be an $\varepsilon$-regular image. Let $f$ be a $k$-face of $\mathbb{F}^{n}$ (with $n-3 \leq k \leq n-1)$.

(i) If $\mathrm{Card}^{-}\left(f_{1}^{\uparrow+}\right) \geq \delta_{\varepsilon}(n-k)$, then $\mu(f)=1$.

(ii) If $\mu(f)=1$, then $\operatorname{Card}^{+}\left(f_{1}^{\uparrow+}\right) \geq \delta_{\varepsilon}(n-k)$. 
Table 2. Necessary and sufficient conditions to obtain $\mu(f)=1$ (see Corollary 1 )

\begin{tabular}{|c|c|c|c|c|}
\hline \multicolumn{5}{|c|}{$\operatorname{dim}(f)=n-2$} \\
\hline$\varepsilon$ & $(\ldots, 1,1)$ & $(\ldots,-1,1)$ & $(\ldots, 1,-1)$ & $(\ldots,-1,-1)$ \\
\hline$C_{\varepsilon}$ & $\operatorname{Card}\left(f_{1}^{\uparrow+}\right) \geq 1$ & $\operatorname{Card}\left(f_{1}^{\uparrow+}\right) \geq 2$ & $\operatorname{Card}\left(f_{1}^{\uparrow+}\right) \geq 3$ & $\operatorname{Card}\left(f_{1}^{\uparrow+}\right) \geq 4$ \\
\hline
\end{tabular}

\begin{tabular}{|c|c|c|l|c|}
\hline \multicolumn{5}{|c|}{$\operatorname{dim}(f)=n-3$} \\
\hline$\varepsilon$ & $(\ldots, 1,1,1)$ & $(\ldots,-1,1,1)$ & $(\ldots, 1,-1,1)$ & $(\ldots,-1,-1,1)$ \\
\hline$C_{\varepsilon}$ & $\operatorname{Card}\left(f_{1}^{\uparrow+}\right) \geq 1$ & $\operatorname{Card}\left(f_{1}^{\uparrow+}\right) \geq 2$ & $\operatorname{Card}\left(f_{1}^{\uparrow+}\right) \geq 3$ & $\begin{array}{c}\operatorname{Card}\left(f_{1}^{\uparrow+}\right) \geq 4 \\
f_{1}^{\uparrow+} \text { not a trihedron }\end{array}$ \\
\hline$\varepsilon$ & $(\ldots, 1,1,-1)$ & $(\ldots,-1,1,-1)$ & $(\ldots, 1,-1,-1)$ & $(\ldots,-1,-1,-1)$ \\
\hline$C_{\varepsilon}$ & $\begin{array}{c}\text { Card }\left(f_{1}^{\uparrow+}\right) \geq 5 \\
\text { or } f_{1}^{\uparrow+} \text { a trihedron }\end{array}$ & $\operatorname{Card}\left(f_{1}^{\uparrow+}\right) \geq 6$ & $\operatorname{Card}\left(f_{1}^{\uparrow+}\right) \geq 7$ & $\operatorname{Card}\left(f_{1}^{\uparrow+}\right)=8$ \\
\hline
\end{tabular}

From Proposition 5 and Definition 1 (needed when $f_{1}^{\uparrow+}$ is a trihedron), we derive the following corollary.

Corollary 1. Let $\mu: \mathbb{F}^{n} \rightarrow\{0,1\}$ be an $\varepsilon$-regular image. Let $f$ be a $k$-face of $\mathbb{F}^{n}$ (with $n-3 \leq k \leq n-1)$. Then $\mu(f)=1$ iff the set $f_{1}^{\uparrow+}$ satisfies the condition $C_{\varepsilon}$ given in Table 2 .

\subsection{Regular Images and Regular Open/Closed Sets}

The object (resp. the background) of a regular image $\mu: \mathbb{F}^{n} \rightarrow\{0,1\}$, i.e., the set $\mu^{-1}(\{1\})$ (resp. $\left.\mu^{-1}(\{0\})\right)$ is topologically regular, i.e., it does not have thin parts nor thin holes (by "thin", we mean of lower dimension than the surrounding space).

Proposition 6. Let $\mu: \mathbb{F}^{n} \rightarrow\{0,1\}$ be an $\varepsilon$-regular image. Let $x \in\{0,1\}$. Then $\left(\mu^{-1}(\{x\})\right)^{\circ}$ is a regular open set and $\left(\mu^{-1}(\{x\})\right)^{\downarrow}$ is a regular closed set.

Corollary 2. Let $\mu: \mathbb{F}^{n} \rightarrow\{0,1\}$ be an $\varepsilon$-regular image. If $\varepsilon=-1$, then $\mu^{-1}(\{1\})$ (resp. $\left.\mu^{-1}(\{0\})\right)$ is a regular open (resp. closed) set. If $\varepsilon=1$, then $\mu^{-1}(\{1\})\left(\right.$ resp. $\left.\mu^{-1}(\{0\})\right)$ is a regular closed (resp. open) set.

\section{Paths and (Digital) Fundamental Groups}

In this section, we study how the functions $\zeta_{\varepsilon}$ behave relatively to the classical notions of path in $\mathbb{Z}^{n}$ and $\mathbb{F}^{n}$. Theorem 2 states that $\zeta_{\varepsilon}$ induces a bijection between the set of the connected components of the object (resp. background) associated to an image $\lambda$ : $\mathbb{Z}^{n} \rightarrow\{0,1\}$, and the set of the connected components of the object (resp. background) associated to the regular image $\mu=\zeta_{\varepsilon}(\lambda)$, the function $\varepsilon$ being chosen with respect to a given pair of adjacencies in $\mathbb{Z}^{n}$. Theorem 3 states that $\zeta_{\varepsilon}$ induces an isomorphism between the digital fundamental group of $\lambda^{-1}(\{1\})$ (resp. $\left.\lambda^{-1}(\{0\})\right)$ and the fundamental group of $\mu^{-1}(\{1\})$ (resp. $\left.\mu^{-1}(\{0\})\right)$. 


\subsection{Background Notions on Paths and Arcs}

The fundamental group of topological spaces. Let $X$ be a topological space. A path $p$ in $X$ is a continuous function $p:[0,1] \rightarrow X$. Two paths $p, q$ in $X$ are equivalent if they have the same extremities (i.e., $p(0)=q(0)$ and $p(1)=q(1)$ ) and $p$ can be continuously deformed to fit $q$, that is if there exists a continuous map $h:[0,1] \times[0,1] \rightarrow X$ such that, for all $t \in[0,1], h(t, 0)=p(t)$ and $h(t, 1)=q(t)$, and, for all $u \in[0,1], h(0, u)=$ $p(0)=q(0)$ and $h(1, u)=p(1)=q(1)$ (the map $h$ is called a path-homotopy). This relation on paths is actually an equivalence relation. We write $[p]$ for the equivalence class of $p$. If $p, q$ are two paths in $X$ such that $p(1)=q(0)$ we can define the product $p \cdot q$ by

$$
(p \cdot q)(t)= \begin{cases}p(2 t) & \text { if } t \in\left[0, \frac{1}{2}\right] \\ q(2 t-1) & \text { if } t \in\left[\frac{1}{2}, 1\right]\end{cases}
$$

This product is well defined on equivalence classes by $[p] \cdot[q]=[p \cdot q]$. Let $x$ be a point of $X$. A loop at $x$ is a path in $X$ which starts and ends at $x$. The product of two loops at $x$ is a loop at $x$ and the set $\pi(X, x)$ of equivalence classes of loops at $x$ is a group for this product. It is called the fundamental group of $X$ (with basepoint $x$ ).

Finite paths in posets. In a poset $X$, a function $f:[0,1] \rightarrow X$ is a step function if there exist finitely many intervals $\left(I_{i}\right)_{i=0}^{r}(r \geq 0)$ such that $f$ is constant on each interval $I_{i}$ and $[0,1]=\bigcup_{i=0}^{r} I_{i}$. A finite path in $X$ is a path in $X$ which is a step function. The sequence $\left(I_{i}\right)_{i=0}^{r}$ is called the intervals sequence of $p$ and the sequence $\left(x_{i}\right)_{i=0}^{r}$ of the values of $f$ the track of $p$. A finite path is regular if there is no singleton in its intervals sequence.

The product of two $\operatorname{arcs}\left(x_{i}\right)_{i=0}^{r}$ and $\left(y_{i}\right)_{i=0}^{s}$ is defined by $\left(x_{i}\right)_{i=0}^{r} .\left(y_{i}\right)_{i=0}^{s}=\left(x_{0}, \ldots, x_{r}\right.$, $\left.y_{1}, \ldots, y_{s}\right)$ provided that $x_{r}=y_{0}$.

An $\operatorname{arc} \chi=\left(x_{i}\right)_{i=0}^{r}(r \geq 2)$ is an elementary stretching (in $\left.X\right)$ of an arc $\chi^{\prime}$ if for some $j \in \llbracket 1, r-1 \rrbracket, \chi^{\prime}=\left(x_{i}\right)_{i=0, i \neq j}^{r}$ or $\left(x_{j-1}=x_{j+1}\right.$ and $\left.\chi^{\prime}=\left(x_{i}\right)_{i=0, i \neq j-1, i \neq j}^{r}\right)$. An $\operatorname{arc} \chi$ is a deformation (in $X$ ) of an arc $\chi^{\prime}$ if there exists a sequence $\left(\chi_{i}\right)_{i=0}^{s}$ of arcs in $X$ such that $\chi_{0}=\chi, \chi_{s}=\chi^{\prime}$ and for any $i \in \llbracket 1, s \rrbracket$, either $\chi_{i}$ is an elementary stretching of $\chi_{i-1}$ or $\chi_{i-1}$ is an elementary stretching in $X$ of $\chi_{i}$.

Let $x$ be a point in $X$. "Being a deformation or equal" is an equivalence relation in the set of $\operatorname{arcs}$ in $X$ from $x$ to $x$. The set of equivalence classes, denoted by $\rho(X, x)$, is a group for the arc product.

Theorem $1([\overline{\mathbf{1 8}}])$. Let $x \in X$. The fundamental group $\pi(X, x)$ of $X$ with basepoint $x$ is isomorphic to the group $\rho(X, x)$.

The digital fundamental group of $\mathbb{Z}^{n}$. A discrete analogue of the concept of fundamental group has been proposed in digital topology [10]. Let $n \in\{2,3\}$ and $X \subseteq \mathbb{Z}^{n}$. The definition of the product for digital paths is straightforward but not so is the notion of equivalence between digital paths or loops. Two paths in $X$ with same extremities are directly equivalent (in $X$ ) if they differ only in a unit lattice cube of $\mathbb{Z}^{n}$ provided that, if $n=3$ and the pair of adjacencies is $(6,26)$, the cube must not contain two diametrically opposite points not in $X$. Finally, two paths in $X, p_{0}, p_{t}(t \geq 0)$, with same extremities are equivalent (in $X$ ) if there is a sequence $\left(p_{i}\right)_{i=0}^{t}$ of paths in $X$ such that, for all $i \in \llbracket 1, t \rrbracket, p_{i}$ is directly equivalent in $X$ to $p_{i-1}$. 


\subsection{Mapping Paths in $\mathbb{Z}^{n}$ onto Arcs in $\mathbb{\mathbb { N }}^{n}$}

Let $\chi$ and $\chi^{\prime}$ be two arcs in $\mathbb{F}^{n}$. We write $\chi \leqslant \chi^{\prime}$ if there exist two paths $p \leqslant p^{\prime}$ in $\mathbb{F}^{n}$ whose tracks are $\chi$ and $\chi^{\prime}$ (all paths in $\mathbb{F}^{n}$ considered in the sequel are regular finite paths).

Definition 2. Let $\omega$ be an adjacency relation on $\mathbb{Z}^{n}$ and $\gamma=\left(p_{i}\right)_{i=0}^{r}(r \geq 0)$ be an $\omega$ path in $\mathbb{Z}^{n}$, given in its reduced form $\left(p_{i} \neq p_{i-1}\right.$ for all $\left.i \in \llbracket 1, r \rrbracket\right)$. We define the arc $\zeta(\gamma)$ in $\mathbb{F}^{n}$ by $\zeta(\gamma)=\left(q_{j}\right)_{j=0}^{2 r}$ with $q_{j}=\dot{p}_{\frac{j}{2}}$ if $j$ is even and $q_{j}=q_{j-1} \cap q_{j+1}$ if $j$ is odd, for all $j \in \llbracket 0,2 r \rrbracket$.

It is obvious that the sequence of faces $\zeta(\gamma)$ defined above is actually an arc in $\mathbb{F}^{n}$ which is itself the track of a regular finite path in $\mathbb{F}^{n}[18]$.

The following proposition states that $\zeta$ associates to a path in the object (resp. in the background), of a digital image $\lambda$, an arc in the object (resp. in the background) of the complex image $\zeta_{\varepsilon}(\lambda)$ under the condition that the connectivity function $\varepsilon$ has been well chosen. The main consequence of this proposition is that the images of the connected components of the digital object (resp. background) are included in the connected components of the image of the object (resp. background).

Proposition 7. Let $(\alpha, \beta)$ be a pair of adjacencies on $\mathbb{Z}^{n}$. Let $\varepsilon$ be the connectivity function associated to $(\alpha, \beta)$. Let $x \in\{0,1\}$. Let $\omega=\alpha$ if $x=1$ and $\omega=\beta$ if $x=0$. Let $\lambda: \mathbb{Z}^{n} \rightarrow\{0,1\}$ be an image in $\mathbb{Z}^{n}$ and $\mu=\zeta_{\varepsilon}(\lambda)$ be the corresponding image in $\mathbb{F}^{n}$. Let $\gamma=\left(p_{i}\right)_{i=0}^{r}(r \geq 0)$ be an $\omega$-path in $\lambda^{-1}(\{x\})$. Then, $\zeta(\gamma)$ is an arc in $\mu^{-1}(\{x\})$.

The following proposition is straightforward.

Proposition 8. Let $\omega$ be an adjacency relation on $\mathbb{Z}^{n}$. The corresponding function $\zeta$ is a homomorphism for the paths product and the arc product: for all $\omega$-paths $\gamma, \gamma^{\prime} \in \mathbb{Z}^{n}$, $\zeta\left(\gamma \cdot \gamma^{\prime}\right)=\zeta(\gamma) \cdot \zeta\left(\gamma^{\prime}\right)$.

The injectivity of $\zeta$ is obvious since two distinct $n$-xels $a, b \in \mathbb{Z}^{n}$ are associated to distinct facets $\dot{a}, \dot{b} \in \mathbb{F}^{n}$. Proposition 9 establishes the surjectivity up to deformations: any $\operatorname{arc} \chi$ from $\dot{a}$ to $\dot{b}$ in an object (resp. in the background) of the complex image is the deformation of an $\operatorname{arc} \zeta(\gamma)$ for some path $\gamma$ from $a$ to $b$ of the object (resp. background) of its associated digital image (if the complex image is associated to such a digital image). Proposition 9 is illustrated by Figure 6 .

Proposition 9. Let $(\alpha, \beta)$ be a pair of adjacencies on $\mathbb{Z}^{n}$. Let $\varepsilon$ be the connectivity function associated to $(\alpha, \beta)$. Let $x \in\{0,1\}$. Let $\omega=\alpha$ if $x=1$ and $\omega=\beta$ if $x=0$. Let $\lambda: \mathbb{Z}^{n} \rightarrow\{0,1\}$ be an image in $\mathbb{Z}^{n}$ and $\mu=\zeta_{\varepsilon}(\lambda)$ be the corresponding image in $\mathbb{F}^{n}$. Let $a, b \in \mathbb{Z}^{n}$. Let $\chi$ be an arc from the facet $\dot{a}$ to the facet $\dot{b}$ in $\mu^{-1}(\{x\})$. Then, there exists an $\omega$-path $\gamma$ from a to $b$ in $\lambda^{-1}(\{x\})$ such that $\zeta(\gamma)$ is a deformation in $\mu^{-1}(\{x\})$ of $\chi$.

Theorem 2. Let $(\alpha, \beta)$ be a pair of adjacencies in $\mathbb{Z}^{n}$. Let $\varepsilon$ be the connectivity function associated to $(\alpha, \beta)$. Let $\lambda: \mathbb{Z}^{n} \rightarrow\{0,1\}$ be a digital image and $\zeta_{\varepsilon}(\lambda)=\mu: \mathbb{F}^{n} \rightarrow$ $\{0,1\}$ be the corresponding complex image. Let $x \in\{0,1\}$. Then the function $\zeta_{\varepsilon}$ induces a one-to-one correspondence between the connected components of $\lambda^{-1}(\{x\})$ and the connected components of $\mu^{-1}(\{x\})$. 


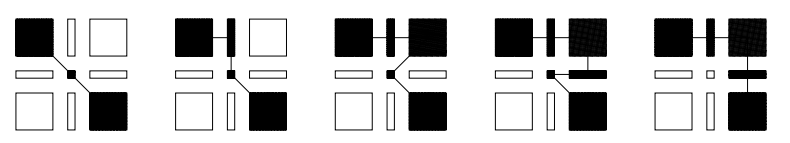

Fig. 6. From an $\operatorname{arc} \mu$ to an $\operatorname{arc} \zeta(\gamma)$ in a (-1)-regular image (the 9 points are in $\mu^{-1}(x)$ )

\subsection{Fundamental Groups}

The aim of this section is to compare the digital fundamental group defined by Kong [10] for subsets of $\mathbb{Z}^{n}, n \in\{2,3\}$, with the fundamental group of subspaces of $\mathbb{F}^{n}$. Thanks to Theorem 1 , we can use arcs as well as paths in $\mathbb{F}^{n}$ in order to perform this comparison.

Proposition 10. Let $(\alpha, \beta)$ be a pair of adjacencies on $\mathbb{Z}^{n}$. Let $\varepsilon$ be the connectivity function associated to $(\alpha, \beta)$. Let $\lambda: \mathbb{Z}^{n} \rightarrow\{0,1\}$ be a digital image and $\zeta_{\varepsilon}(\lambda)=\mu$ : $\mathbb{F}^{n} \rightarrow\{0,1\}$ be the corresponding complex image. Let $x \in\{0,1\}$. Let $\omega=\alpha$ if $x=1$ and $\omega=\beta$ if $x=0$. Two $\omega$-paths in $\lambda^{-1}(\{x\}), \gamma$ and $\gamma^{\prime}$, are equivalent in $\lambda^{-1}(\{x\})$ iff the arc $\zeta(\gamma)$ is equal to or is a deformation in $\mu^{-1}(\{x\})$ of the arc $\zeta\left(\gamma^{\prime}\right)$.

Let $a \in \lambda^{-1}(\{x\})$. Let $\pi_{D}\left(\lambda^{-1}(\{x\}), a\right)$ be the digital fundamental group of $\lambda^{-1}(\{x\})$ with basepoint $a$ and $\rho\left(\mu^{-1}(\{x\}), \dot{a}\right)$ be the group of $\operatorname{arcs}$ in $\mu^{-1}(x)$ from $\dot{a}$ to $\dot{a}$, up to deformations.

From Propositions 7 and 10 , we know that the function $\dot{\zeta}$ defined by

$$
\mid \begin{array}{ccc}
\dot{\zeta}: & \pi_{D}\left(\lambda^{-1}(x), a\right) & \rightarrow \rho\left(\mu^{-1}(x), \dot{a}\right) \\
{[\gamma]} & \mapsto \quad[\zeta(\gamma)]
\end{array}
$$

where $[y]$ denotes the equivalence class of $y$ (for the equivalence relation on digital paths of $\mathbb{Z}^{n}$ on the left side and for the deformation on arcs of $\mathbb{F}^{n}$ on the right side), is well-defined. Proposition 8 then states that $\dot{\zeta}$ is a morphism. Propositions 9 and 10 give the surjectivity and the the injectivity of $\dot{\zeta}$, respectively. We conclude that the groups $\pi_{D}\left(\lambda^{-1}(\{x\}), a\right)$ and $\rho\left(\mu^{-1}(\{x\}), \dot{a}\right)$ are isomorphic and, since $\rho\left(\mu^{-1}(\{x\}), \dot{a}\right)$ and $\pi\left(\mu^{-1}(\{x\}), \dot{a}\right)$ are isomorphic (Theorem 1 ), the following theorem holds.

Theorem 3. Let $(\alpha, \beta)$ be a pair of adjacencies on $\mathbb{Z}^{n}$. Let $\varepsilon$ be the connectivity function associated to $(\alpha, \beta)$. Let $\lambda: \mathbb{Z}^{n} \rightarrow\{0,1\}$ be an image in $\mathbb{Z}^{n}$ and $\mu=\zeta_{\varepsilon}(\lambda)$ be the corresponding image in $\mathbb{F}^{n}$. For any $a \in \lambda^{-1}(\{x\})$, the digital fundamental group of $\lambda^{-1}(\{x\})$ with basepoint a is isomorphic to the fundamental group of the poset $\left(\mu^{-1}(\{x\}), \subseteq\right)$ with base point $\dot{a}$.

\section{Conclusion}

In this article, a modus operandi has been proposed to embed digital binary images, equipped with a pair of standard adjacencies, in the space of cubical complexes. In particular, it has been proved that it preserves the connected components of both object and background and, more generally, preserves also the (digital) fundamental groups.

These results, associated to those of [18], justify the soundness of all the contributions previously devoted to design homotopy type-preserving binary image processing 
methods, especially concerning the correctness of their behaviour with respect to the "continuous" topology of the handled digital objects. They also permit to establish links between image processing methods developed either in classical digital spaces $\left(\mathbb{Z}^{n}\right)$ or cubical complexes $\left(\mathbb{F}^{n}\right)$, and to potentially unify some of them. An extended version of this work (with proofs of the propositions and theorems) will be proposed soon [17].

\section{References}

1. Aktouf, Z., Bertrand, G., Perroton, L.: A three-dimensional holes closing algorithm. Pattern Recognition Letters 23(5), 523-531 (2002)

2. Ayala, R., Domínguez, E., Francés, A.R., Quintero, A.: Digital lighting functions. In: Ahronovitz, E. (ed.) DGCI 1997. LNCS, vol. 1347, pp. 139-150. Springer, Heidelberg (1997)

3. Ayala, R., Domínguez, E., Francés, A.R., Quintero, A.: Digital homotopy with obstacles. Discrete Applied Mathematics 139(1-3), 5-30 (2004)

4. Bertrand, G.: New notions for discrete topology. In: Bertrand, G., Couprie, M., Perroton, L. (eds.) DGCI 1999. LNCS, vol. 1568, pp. 218-228. Springer, Heidelberg (1999)

5. Bertrand, G., Couprie, M.: A model for digital topology. In: Bertrand, G., Couprie, M., Perroton, L. (eds.) DGCI 1999. LNCS, vol. 1568, pp. 229-241. Springer, Heidelberg (1999)

6. Bertrand, G., Malandain, G.: A new characterization of three-dimensional simple points. Pattern Recognition Letters 15(2), 169-175 (1994)

7. Faisan, S., Passat, N., Noblet, V., Chabrier, R., Meyer, C.: Topology-preserving warping of binary images according to one-to-one mappings. IEEE Transactions on Image Processing (to appear)

8. Gonzalez-Diaz, R., Jimenez, M.J., Medrano, B.: Well-composed cell complexes (November 2010), http://personal.us.es/majiro/ctic19.pdf communication at CTIC 2010

9. Khalimsky, E., Kopperman, R., Meyer, P.R.: Computer graphics and connected topologies on finite ordered sets. Topology and its Applications 36(1), 1-17 (1990)

10. Kong, T.Y.: A digital fundamental group. Computers and Graphics 13(2), 159-166 (1989)

11. Kong, T.Y., Roscoe, A.W.: A theory of binary digital pictures. Computer Vision, Graphics and Image Processing 32(2), 221-243 (1985)

12. Kong, T.Y.: Topology-preserving deletion of 1's from 2-, 3- and 4-dimensional binary images. In: Ahronovitz, E. (ed.) DGCI 1997. LNCS, vol. 1347, pp. 3-18. Springer, Heidelberg (1997)

13. Kong, T.Y., Rosenfeld, A.: Digital topology: introduction and survey. Computer Vision, Graphics and Image Processing 48(3), 357-393 (1989)

14. Kovalevsky, V.A.: Finite topology as applied to image analysis. Computer Vision, Graphics, and Image Processing 46(2), 141-161 (1989)

15. Lachaud, J.O., Montanvert, A.: Continuous analogs of digital boundaries: A topological approach to iso-surfaces. Graphical Models and Image Processing 62, 129-164 (2000)

16. Latecki, L.J.: 3d well-composed pictures. Graph. Models Image Process. 59(3), 164-172 (1997)

17. Mazo, L., Passat, N., Couprie, M., Ronse, C.: Digital imaging: A unified topological framework. Tech. Rep. hal-00512270, Université Paris-Est (2010), http://hal .archives-ouvertes.fr/hal-00512270/fr/

18. Mazo, L., Passat, N., Couprie, M., Ronse, C.: Paths, homotopy and reduction in digital images. Acta Applicandae Mathematicae 113(2), 167-193 (2011)

19. Rosenfeld, A.: Connectivity in digital pictures. Journal of the Association for Computer Machinery 17(1), 146-160 (1970) 